\title{
Open shells in reduced-density-matrix-functional theory
}

\author{
N. N. Lathiotakis, ${ }^{1}$ N. Helbig, ${ }^{1}$ and E. K. U. Gross ${ }^{1}$ \\ ${ }^{1}$ Institut für Theoretische Physik, Freie Universität Berlin, Arnimallee 14, D-14195 Berlin, Germany
}

\begin{abstract}
Reduced-density-matrix-functional theory is applied to open-shell systems. We introduce a spinrestricted formulation by appropriately expressing approximate correlation-energy functionals in terms of spin-dependent occupation numbers and spin-independent natural orbitals. We demonstrate that the additional constraint of total-spin conservation is indispensable for the proper treatment of open-shell systems. The formalism is applied to the first-row open-shell atoms. The obtained ground-state energies are in very good agreement with the exact values as well as other state of the art quantum chemistry calculations.
\end{abstract}

PACS numbers: 31.25.-v,31.25.Eb,31.15.Ne,31.15.Ew

The proper description of electronic correlation is the central problem in theoretical material science. Density functional theory (DFT) deals with this problem by considering the electronic density as the fundamental variable. DFT is built upon the Hohenberg-Kohn theorem [1] which ensures that the expectation value of every observable, including the total energy, is a unique functional of the electronic ground-state density. The many-electron problem is then mapped onto an auxiliary, non-interacting system, the so-called Kohn-Sham system [2] whose density reproduces the interacting density. In practice, the so-called exchange and correlation part of the Kohn-Sham potential needs to be approximated. DFT is tremendously successful in describing properties of real materials and that success is proved by its general acceptance as one of the major tools in exploring theoretically the world of real materials. Nevertheless, there are certain materials, termed collectively as highly correlated materials, for which the results of DFT, at least with standard functionals for exchange and correlation, deviate significantly from the experimental values.

Reduced-density-matrix-functional theory (RDMFT) is an alternative way to deal with the many-electron problem. It is based on Gilbert's theorem [3], which guarantees that the expectation value of any observable of the system in the ground-state is a unique functional of the one-body reduced-density-matrix (1-RDM)

$$
\gamma\left(\mathbf{r}, \mathbf{r}^{\prime}\right)=N \int d^{3} \mathbf{r}_{2} \cdots d^{3} \mathbf{r}_{N} \Psi^{*}\left(\mathbf{r}^{\prime}, \mathbf{r}_{2} \cdots \mathbf{r}_{N}\right) \Psi\left(\mathbf{r}, \mathbf{r}_{2} \cdots \mathbf{r}_{N}\right)
$$

where $\Psi$ is the many-body ground-state wavefunction. The advantage of this approach, compared to DFT, is that the exact many-body kinetic energy is easily expressed in terms of $\gamma$. Although the properties of 1-RDM functionals had been the subject of theoretical studies for a long time [3, 4, 5, 6], there were few practical applications of RDMFT until recently.

We emphasize that it is not possible to construct a Kohn-Sham-like independent electron scheme in RDMFT: Due to its non-idempotency, the 1-RDM of an interacting system cannot be reproduced by a noninteracting system because the latter always has an idempotent 1-RDM. This is reflected in the eigenvalues of the 1-RDM which are equal to zero or one for a noninteracting system, while in the interacting case some of them are fractional.

The first implicit functional of the 1-RDM was introduced in 1984 by Müller [6]. This functional was later derived from modeling the correlation hole, by Buijse and Baerends [7]. Goedecker and Umrigar [8] introduced a self-interaction correction to this functional. Other functionals have been proposed more recently $9,10,11,12$, 13, 14. Implicit 1-RDM functionals depend explicitly on the so-called natural orbitals $\varphi_{a}$ and the corresponding occupation numbers $n_{a}$ which are defined as the eigenfunctions and the eigenvalues of the 1-RDM

$$
\int d \mathbf{r}^{\prime 3} \gamma\left(\mathbf{r}, \mathbf{r}^{\prime}\right) \varphi_{a}\left(\mathbf{r}^{\prime}\right)=n_{a} \varphi_{a}(\mathbf{r})
$$

The total energy can be minimized with respect to the natural orbitals and the occupation numbers, instead of the 1-RDM itself, by considering the appropriate subsidiary conditions, namely the conservation of the total number of electrons, the $N$-representability constraint and the orbital orthonormality. Goedecker and Umrigar 8] calculated the correlation energy of small atomic systems including the open-shell Lithium and Carbon atom. Despite their simplicity, 1-RDM functionals are able to provide a good approximation of the correlation energy of small systems [7, 8, 9, 10, 11, 12, 15, 16]. Generally, the correlation energy of finite systems is improved by removing the self-interaction terms 15, 16]. However the correct dissociation limit of the $\mathrm{H}_{2}$ molecule is found only if self-interaction is retained $[\mathbf{7}$, 15, 16.

In the present work, we demonstrate how natural orbital functionals in RDMFT can be applied to open-shell systems. For that purpose, we employ the Müller functional [6] with the self-interaction correction proposed by Goedecker and Umrigar [8]. Our formulation is spinrestricted, i.e. we have spin-dependent occupation numbers but spin-independent natural orbitals. The advantage of this approach is that particular spin configu- 
rations are prescribed, in the same manner as in the restricted open-shell Hartree-Fock (ROHF) method [17]. We discuss the necessity of a spin-dependent constraint for the conservation of both the total number of electrons as well as the spin.

We start with the 1-RDM functional described in Refs. [6, 7, 8], where we explicitly include the full spin dependence in the occupation numbers and the natural orbitals. We refer to this as the spin-unrestricted functional that reads

$$
\begin{aligned}
E=\sum_{a, \sigma} n_{a}^{\sigma} h_{a a}^{\sigma}+\frac{1}{2} & \sum_{a b, \sigma \sigma^{\prime}} n_{a}^{\sigma} n_{b}^{\sigma^{\prime}}\left(1-\delta_{a b} \delta_{\sigma \sigma^{\prime}}\right) J_{a b}^{\sigma \sigma^{\prime}} \\
& -\frac{1}{2} \sum_{a b, \sigma} \sqrt{n_{a}^{\sigma} n_{b}^{\sigma}}\left(1-\delta_{a b}\right) K_{a b}^{\sigma}
\end{aligned}
$$

with $n_{a}^{\sigma}$ being the spin-dependent occupation numbers and $h_{a a}^{\sigma}, J_{a b}^{\sigma \sigma^{\prime}}$ and $K_{a b}^{\sigma}$ the one- and two-electron integrals for the natural orbitals $\varphi_{a \sigma}$

$$
\begin{aligned}
h_{a a}^{(\sigma)} & =\int d^{3} \mathbf{r} \varphi_{a \sigma}^{*}(\mathbf{r})\left[-\frac{\nabla^{2}}{2}+V_{e x t}(\mathbf{r})\right] \varphi_{a \sigma}(\mathbf{r}) \\
J_{a b}^{\sigma \sigma^{\prime}} & =\iint d^{3} \mathbf{r} d^{3} \mathbf{r}^{\prime} \frac{\varphi_{a \sigma}^{*}(\mathbf{r}) \varphi_{a \sigma}(\mathbf{r}) \varphi_{b \sigma^{\prime}}\left(\mathbf{r}^{\prime}\right) \varphi_{b \sigma^{\prime}}^{*}\left(\mathbf{r}^{\prime}\right)}{\left|\mathbf{r}-\mathbf{r}^{\prime}\right|} \\
K_{a b}^{\sigma} & =\iint d^{3} \mathbf{r} d^{3} \mathbf{r}^{\prime} \frac{\varphi_{a \sigma}^{*}(\mathbf{r}) \varphi_{b \sigma}(\mathbf{r}) \varphi_{a \sigma}\left(\mathbf{r}^{\prime}\right) \varphi_{b \sigma}^{*}\left(\mathbf{r}^{\prime}\right)}{\left|\mathbf{r}-\mathbf{r}^{\prime}\right|}
\end{aligned}
$$

In the above expression, $V_{\text {ext }}(\mathbf{r})$ is the external potential, i.e. the ionic potential for atomic and molecular systems. The Kronecker $\delta$ s are inserted in Eq. (3) in order to exclude explicitly the self-interaction terms 8 ].

We now concentrate on the spin-restricted case by assuming spin-independent orbitals but still spindependent occupation numbers. Then the expression (3) reduces to

$$
\begin{aligned}
E & =\sum_{a}\left(n_{a}^{\uparrow}+n_{a}^{\downarrow}\right) h_{a a}+ \\
\frac{1}{2} \sum_{a b} & {\left[\left(n_{a}^{\uparrow} n_{b}^{\uparrow}+n_{a}^{\downarrow} n_{b}^{\downarrow}\right)\left(1-\delta_{a b}\right)+\left(n_{a}^{\uparrow} n_{b}^{\downarrow}+n_{a}^{\downarrow} n_{b}^{\uparrow}\right)\right] J_{a b} } \\
& -\frac{1}{2} \sum_{a b}\left[\left(\sqrt{n_{a}^{\uparrow} n_{b}^{\uparrow}}+\sqrt{n_{a}^{\downarrow} n_{b}^{\downarrow}}\right)\left(1-\delta_{a b}\right)\right] K_{a b} \cdot
\end{aligned}
$$

In this equation, the integrals $h_{a a}, J_{a b}$ and $K_{a b}$ are analogous to those in Eq. (4) but for spin-independent orbitals $\varphi_{a}$. This is the functional we employ in this work. It can be viewed as a generalization of the ROHF functional in the sense that it reduces to the ROHF functional if all the occupation numbers are either zero or one. Although the formulation in this paper refers to the specific functional given in Eq. (5), the generalization to different functionals of similar kind [9] is easily achieved by replacing the square roots in the exchange-like term in Eq. (5) by the appropriate functions $f\left(n_{a}^{\uparrow}, n_{b}^{\uparrow}\right), f\left(n_{a}^{\downarrow}, n_{b}^{\downarrow}\right)$.
Driven by physical requirements, Cioslowski et al 18$]$ have derived a list of criteria that 1-RDM functionals should fulfill. In addition, they examined whether functionals of the Müller-type satisfy these criteria. Our open-shell version (5) of the self-interaction-corrected Müller functional satisfies the same criteria as the original Müller functional [6] with the self-interaction correction of Goedecker and Umrigar [8].

We now discuss the subsidiary conditions that have to be enforced in the minimization procedure of functionals like the one given by Eq. (5). Since the occupation numbers are spin-dependent we face a dilemma concerning the conservation of the total spin. More specifically we have the following two options: (i) to use one constraint for the conservation of the total number of electrons. This introduces a single Lagrange multiplier, the chemical potential $\mu$. In that way, we unfortunately allow for charge transfer from one spin channel to the other, usually from the majority to the minority spin and the total spin is not preserved, or (ii) to use an extra constraint for the conservation of the total spin, making the minimization more restrictive. In practice, we use two different constraints for the spin-up and the spin-down electrons. In that way, we introduce two Lagrange multipliers, or, in other words, a spin-dependent chemical potential. It is one of the goals of the present work to assess these two different ways of minimizing the energy functional. Of course, the above dilemma applies only to open-shell systems. It is expected that the first option, being less restrictive, leads to a lower total energy. However, since RDMFT (like DFT) is not variational, a lower energy is not necessarily better. Hence, it is not a priori clear whether enforcing the spin conservation constraint in addition to the particle number conservation will improve or worsen the energy.

Additionally, as in the case of closed-shell systems, we have to include two further subsidiary conditions. The first is the orbital orthonormality. Unfortunately, all 1RDM functionals are not invariant under unitary transformation of the natural orbitals. This leads to a complex minimization problem which consists of orbital dependent Fock-like operators and off-diagonal Lagrange multipliers. The equations we have to solve in order to find the orbital solution for fixed but fractional occupation numbers have the form

$$
\begin{aligned}
F^{(a)}(\mathbf{r}) \varphi_{a}(\mathbf{r}) & =\sum_{b} \epsilon_{a b} \varphi_{b}(\mathbf{r}) \\
\epsilon_{b a} & =\epsilon_{a b}
\end{aligned}
$$

where $F^{(a)}$ is analogous to the Fock matrix in HartreeFock theory but in this case orbital-index dependent, and $\epsilon_{a b}$ are the non-diagonal Lagrange multipliers, which should be Hermitian according to the second equation. 
The orbital-index dependent operator $F^{(a)}$ is

$$
\begin{aligned}
& F^{(a)}(\mathbf{r})=\left(n_{a}^{\uparrow}+n_{a}^{\downarrow}\right) h(\mathbf{r})+ \\
& \sum_{b}\left[\left(n_{a}^{\uparrow} n_{b}^{\uparrow}+n_{a}^{\downarrow} n_{b}^{\downarrow}\right)\left(1-\delta_{a b}\right)+\left(n_{a}^{\uparrow} n_{b}^{\downarrow}+n_{a}^{\downarrow} n_{b}^{\uparrow}\right)\right] \mathcal{J}_{b}(\mathbf{r}) \\
& \quad-\sum_{a b}\left[\left(\sqrt{n_{a}^{\uparrow} n_{b}^{\uparrow}}+\sqrt{n_{a}^{\downarrow} n_{b}^{\downarrow}}\right)\left(1-\delta_{a b}\right)\right] \mathcal{K}_{b}(\mathbf{r}), \quad(7)
\end{aligned}
$$

where

$$
\begin{aligned}
h(\mathbf{r}) & =-\frac{\nabla^{2}}{2}+V_{e x t}(\mathbf{r}), \\
\mathcal{J}_{b}(\mathbf{r}) & =\int d \mathbf{r}^{\prime} \frac{\varphi_{b}^{*}\left(\mathbf{r}^{\prime}\right) \varphi_{b}\left(\mathbf{r}^{\prime}\right)}{\left|\mathbf{r}-\mathbf{r}^{\prime}\right|} \\
\mathcal{K}_{b}(\mathbf{r}) \varphi_{a}(\mathbf{r}) & =\int d \mathbf{r}^{\prime} \frac{\varphi_{b}^{*}\left(\mathbf{r}^{\prime}\right) \varphi_{a}\left(\mathbf{r}^{\prime}\right)}{\left|\mathbf{r}-\mathbf{r}^{\prime}\right|} \varphi_{b}(\mathbf{r}) .
\end{aligned}
$$

A similar problem with an orbital-index dependent operator exists in the self-interaction-correction method 19 , 20] of DFT and to a lesser extent in the ROHF method 17, 21]. Finally, the second of the subsidiary conditions, known as the N-representability constraint, restricts the occupation numbers to lie between zero and one, $0 \leq n_{a}^{\sigma} \leq 1$ and guarantees that the 1-RDM corresponds to an $N$-body wave function.

Our implementation uses the GAMESS quantum chemistry program 23 for the calculation of the onebody and two-body integrals as well as for providing the Hartree-Fock solution which we choose as the starting point of our minimization procedure. The minimizations with respect to both the occupation numbers and the natural orbitals are performed with a conjugate gradient procedure. For the optimization with respect to the orbitals, we adopted a procedure similar to the one described in Refs. 20, 22]. We calculated the total energies of the first-row open-shell atoms, i.e. Li, B, C, N, O, and F. For all these elements we used the cc-PVQZ Gaussianbasis set 24.

In Figure 1 we show the convergence of the total energy as a function of the number of natural orbitals included in the calculation. As it can be seen, 30-40 natural orbitals are typically required for full convergence. In Table【 we list the total energies for the open-shell systems as well as the He and Be closed-shell atoms for completeness. Comparing the total energies it becomes apparent that forcing or relaxing the constraint of spin conservation is extremely important for open-shell systems. The energy differences between the two minimization procedures are of the order of $10 \mathrm{mH}$ with the spin-conserving constraint giving results much closer to the exact ones. Relaxing the constraint results in a charge transfer from one spin to the other which is of the order of 0.05-0.1 electrons. This charge transfer increases with the number of natural orbitals included in the minimization procedure which is the reason for the increase in the energy difference between the two procedures with the number of
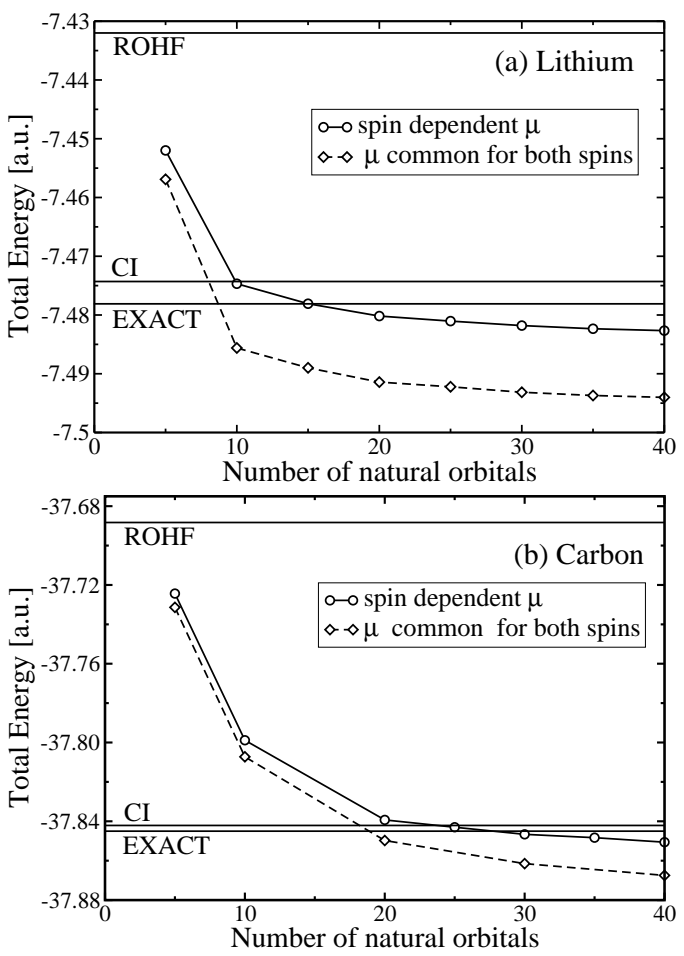

FIG. 1: The convergence of the total energy with the number of natural orbitals included in the minimization procedure for Lithium and Carbon atoms. The values of the ROHF method as well as the CI and exact values are shown as horizontal lines.

naturals orbitals seen in Fig. 10 Interestingly, for the last two open-shell elements, i.e. O and F, where the 2 p shell is more than half filled, the energy difference between the two minimization procedures is smallest.

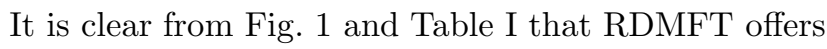
a very good approximation for the correlation energy for all, closed and open-shell, atomic systems we studied. Despite its simplicity, the functional we used produces results in very good agreement with state of the art, but computationally much more expensive, methods like CI. However, the systematic trend of this particular functional is to overestimate slightly the correlation energy for all the open-shell systems studied. To give a fair credit to RDMFT one has to take into account that this is one of the very first 1-RDM functionals that has been exploited in a minimization procedure, in contrast to DFT functionals that have been heavily used and tuned for decades.

In Table we quote the different values of the chemical potential for the majority and the minority spin as well as the common value of it in the case of relaxing the constraint of spin conservation. Clearly, the values are significantly different. Interestingly, for $\mathrm{B}$ and $\mathrm{C}$ with the p-shell less than half filled, but also for $\mathrm{N}$ with half filled p-shell, the majority $\mu^{\uparrow}$ is larger than the minority $\mu^{\downarrow}$. This is a consequence of the fact that ionization from 


\begin{tabular}{lcccc}
\hline \hline Atom & \multicolumn{2}{c}{ RDMFT } & QCI & Exact \\
& $\mu^{\uparrow}=\mu^{\downarrow}$ & $\mu^{\downarrow} \neq \mu^{\uparrow}$ & & \\
\hline $\mathrm{He}$ & \multicolumn{2}{c}{2.8978} & 2.9049 & 2.9037 \\
$\mathrm{Li}$ & 7.4940 & 7.4827 & 7.4743 & 7.4781 \\
$\mathrm{Be}$ & \multicolumn{2}{c}{14.6686} & 14.6657 & 14.6674 \\
$\mathrm{~B}$ & 24.6746 & 24.6616 & 24.6515 & 24.6539 \\
$\mathrm{C}$ & 37.8675 & 37.8506 & 37.8421 & 37.8450 \\
$\mathrm{~N}$ & 54.6096 & 54.5965 & 54.5854 & 54.5893 \\
$\mathrm{O}$ & 75.0668 & 75.0671 & 75.0613 & 75.0670 \\
$\mathrm{~F}$ & 99.6951 & 99.6952 & 99.727 & 99.734 \\
\hline $\bar{\Delta}$ & 0.020 & 0.010 & 0.004 & 0.000 \\
\hline \hline
\end{tabular}

TABLE I: Absolute total energies for the first row atoms (in a.u.) with and without enforcing the spin conservation constraint. The QCI 25] and exact 26] values are also listed. $\bar{\Delta}$ is the mean absolute deviation from the exact values (for open-shell systems only).

\begin{tabular}{lcc|cc}
\hline \hline & \multicolumn{2}{c|}{$\begin{array}{c}\text { Forcing spin } \\
\text { conservation }\end{array}$} & \multicolumn{2}{c}{$\begin{array}{c}\text { Relaxing spin } \\
\text { conservation }\end{array}$} \\
& $-\mu^{\uparrow}[$ a.u. $]$ & $-\mu^{\downarrow}[$ a.u. $]$ & $-\mu[$ a.u. $]$ & $\Delta q[\mathrm{e}]$ \\
\hline $\mathrm{He}$ & 0.928 & 0.928 & 0.928 & - \\
$\mathrm{Li}$ & 0.191 & 2.541 & 0.186 & 0.012 \\
$\mathrm{Be}$ & 0.292 & 0.292 & 0.292 & - \\
$\mathrm{B}$ & 0.234 & 0.421 & 0.193 & 0.089 \\
$\mathrm{C}$ & 0.312 & 0.550 & 0.286 & 0.074 \\
$\mathrm{~N}$ & 0.445 & 0.710 & 0.411 & 0.058 \\
$\mathrm{O}$ & 0.474 & 0.404 & 0.418 & 0.075 \\
$\mathrm{~F}$ & 0.570 & 0.527 & 0.510 & 0.060 \\
\hline \hline
\end{tabular}

TABLE II: The values of the spin-dependent chemical potential $\mu^{\uparrow}$ (the majority spin) and $\mu^{\downarrow}$ in the case of enforcing the spin conservation constraint, and the common value of $\mu$ and the charge transfer $\Delta q$ in the case of relaxing that constraint.

the majority is energetically favorable. For the remaining two elements, i.e. $\mathrm{O}$ and $\mathrm{F}$, with more than half filled p-shell this trend is opposite.

In summary, we have presented a systematic application of RDMFT to open-shell systems. We adopted a spin-restricted open-shell treatment and extended 1RDM functionals to include spin-dependent occupation numbers. This formalism has the advantage of allowing the prescription of a specific spin state. In particular, we introduced a spin-dependent chemical potential in order to enforce conservation of the total spin in the minimization procedure, in addition to the conservation of the total number of electrons. We performed calculations for open-shell atoms using the appropriate extension of a standard 1-RDM functional [6, 8], both with and without the spin conservation constraint. We conclude that the inclusion of this constraint is essential for the proper treatment of open-shell systems. Our results for the total energies of the first row open-shell atoms are in very good agreement with state-of-the-art quantum-chemistry calculations. The presented formalism can be easily extended to any 1-RDM functional and therefore opens up the wide range of open-shell systems to the testing of new 1-RDM functionals.

We thank A. Zacarias for helpful suggestions. This work was supported in part by the Deutsche Forschungsgemeischaft within the program SPP 1145 , by the EXCITING Research and Training Network and by the NANOQUANTA Network of Excellence.

[1] P. Hohenberg and W. Kohn, Phys. Rev. 136, B864 (1964).

[2] W. Kohn, L. J. Sham, Phys. Rev. 140, A1133 (1965).

[3] T. L. Gilbert, Phys. Rev. B12, 2111 (1975).

[4] S. M. Valone, J. Chem. Phys. 73, 1344 (1980); 73, 4653 (1980).

[5] G. Zumbach and K. Maschke, J. Chem. Phys. 82, 5604 (1985).

[6] A. M. K. Müller, Phys. Lett. A 105, 446 (1984).

[7] M.A. Buijse, PhD Thesis, Vrije Universiteit Amsterdam (1991); M. A. Buijse, E. J. Baerends, Mol. Phys. 100, 401 (2002).

[8] S. Goedecker, C. J. Umrigar, Phys. Rev. Lett. 81, 866 (1998).

[9] G. Csányi, T. A. Arias, Phys. Rev. B61 (2000) 7348.

[10] G. Csányi, S. Goedecker, T. A. Arias, Phys. Rev. A65 032510 (2002).

[11] K. Yasuda, Phys. Rev. Lett. 88, 053001 (2002).

[12] C. Kollmar, J. Chem. Phys. 121, 11581 (2004).

[13] J. Cioslowski, K. Pernal, M. Buchowiecki, J. Chem. Phys. 119, 6443 (2003).

[14] K. Pernal, J. Cioslowski, J. Chem. Phys. 120, 5987 (2004)

[15] V. N. Staroverov and G. E. Scuseria, J. Chem. Phys. 117, 2489 (2002).

[16] J. M. Herbert, J. E. Harriman, Chem. Phys. Lett. 382, 142 (2003).

[17] C. C. J. Roothaan, Rev. Mod. Phys. 32, 179 (1960).

[18] J. Cioslowski, K. Pernal, P. Ziesche, J. Chem. Phys. 117, 9560 (2002).

[19] A. Svane, Phys. Rev B53, 4275 (1996).

[20] S. Goedecker, C. J. Umrigar, Phys. Rev. A55, 1765 (1997).

[21] K. Hirao and H. Nakatsuji, The J. Chem. Phys. 59, 1457 (1973).

[22] A. J. Cohen, E. J. Baerends, Chem. Phys. Lett. 364, 409 (2002).

[23] M. W. Schmidt, K. K. Baldridge, J. A. Boatz, S. T. Elbert, M. S. Gordon, J. Jensen, S. Koseki, N. Matsunaga, K. A. Nguyen, S. Su, T. L. Windus, M. Dupuis, J. A. Montgomery, J. Comput. Chem. 14, 1347 (1993).

[24] T. H. Dunning, Jr., J. Chem. Phys. 901007 (1989); D. E. Woon, T.H. Dunning, Jr., J. Chem. Phys. 1002975 (1994)

[25] J. A. Montgomery, J. W. Ochterski, G. A. Petersson, J. Chem. Phys. 101, 5900 (1994).

[26] E. R. Davidson, S. A. Hagstrom, S. J. Chakravorty, V. M. Umar, C. Froese Fischer, Phys. Rev. A44, 7071 (1991). 